\title{
Convergence of optimal solutions in control problems for hyperbolic equations
}

\author{
by S. MigóRski (Kraków)
}

\begin{abstract}
A sequence of optimal control problems for systems governed by linear hyperbolic equations with the nonhomogeneous Neumann boundary conditions is considered. The integral cost functionals and the differential operators in the equations depend on the parameter $k$. We deal with the limit behaviour, as $k \rightarrow \infty$, of the sequence of optimal solutions using the notions of $G$ - and $\Gamma$-convergences. The conditions under which this sequence converges to an optimal solution for the limit problem are given.
\end{abstract}

1. Introduction. In this note, we consider the sequence of optimal control problems for systems described by second-order linear hyperbolic equation

$$
\frac{\partial^{2} y}{\partial t^{2}}-\frac{\partial}{\partial x_{i}}\left(a_{i j}^{k}(x, t) \frac{\partial y}{\partial x_{j}}\right)=f
$$

with the Cauchy initial data and the nonhomogeneous Neumann boundary conditions. The parameter $k \in \mathbb{N}$ (index of an element of the sequence) appears in the coefficients of the state equations as well as in the cost functionals which have a general integral form.

Our motivation is mainly related to boundary control problems with homogenization in the state equation (see for example [10]); however, the role of controls is played not only by the boundary functions but also by the forcing term in the equation and the initial functions.

We formulate the control problem in the following way (see e.g. [9] and [2]): find

$$
\min \left\{J_{k}(u, y)+\chi_{\Lambda_{k}}(u, y) \mid(u, y) \in U \times Y\right\},
$$

1991 Mathematics Subject Classification: 49J20, 49J45, 49K40.

Key words and phrases: control problem, hyperbolic equation, $G$-convergence, $\Gamma$-convergence.

This work has been performed as part of Research Project nr 210739101 supported by K.B.N. This paper was completed while the author was visiting Scuola Normale Superiore, Pisa, Italy. 
where $J_{k}: U \times Y \rightarrow \mathbb{R} \cup\{ \pm \infty\}$ are the cost functionals; $U, Y$ are the spaces of control and states, respectively, $\Lambda_{k} \subset U \times Y$ are the sets of admissible pairs $(u, y)$ and $\chi_{\Lambda_{k}}$ denotes the indicator function of $\Lambda_{k}$ (i.e. $\chi_{\Lambda_{k}}=0$ on $\Lambda_{k}$, and $\infty$ elsewhere). This is of course an equivalent formulation of the problem of minimization of $J_{k}$ over the sets $\Lambda_{k}$. The elements which realize the minimum in $(1)_{k}$ are called optimal solutions.

In this paper we consider two problems: (i) we study the existence of optimal solutions for every fixed parameter $k$ and (ii) we investigate the asymptotic behaviour of the sequence of optimal solutions as $k \rightarrow \infty$.

We get an affirmative answer to problem (i) by using the direct method of the calculus of variations. As concerns (ii), our approach is based on an abstract framework given in [2] for characterization of the limits of control problems. The abstract scheme requires the $\Gamma$-convergence of the cost functionals and of the indicator functions of the sets of admissible pairs (see Proposition 4.1 below). This formulation was applied to study control problems for elliptic equations in [2], [14] and for evolution equations in [8], [15], [16].

Here, we give conditions under which the optimal solutions of the sequence $(1)_{k}$ converge to an optimal solution of the limit problem of the same kind. In this way, we extend the earlier results (see Theorem 6.2 of [8] and Lemma 2.2 of [13]) to the class of control problems for hyperbolic equations with the nonhomogeneous Neumann boundary conditions. Such equations are treated by using the transposition method described in [11] and [9]. The $\Gamma$-convergence of the cost functionals is obtained in a similar way to that used in [2], [8], [14] starting with results of [12]. We prove the $\Gamma$-convergence of the sets $\Lambda_{k}$ employing the notion of $G$-convergence introduced in [18] for elliptic operators and extended to parabolic and hyperbolic equations in [3], [4] (for more details we refer to [5], [19], [7], [20], [15]). Finally, we remark that a special case in which $G$-convergence holds is that of homogenization (in the space variable $x$ ), where $a_{i j}^{k}(x, t)=\alpha_{i j}(k x, t)$ for some $\alpha_{i j}(y, t)$ periodic in $y$ (compare for instance [1] and [17]).

The main result of this paper was announced in its preliminary form in $[15]$.

2. Preliminaries. We shall briefly introduce the essential notations and state some results needed in the sequel.

We consider a Gelfand triple of separable Hilbert spaces $V \subset H \subset V^{\prime}$ with continuous, dense and compact embeddings. We denote by $\langle\cdot, \cdot\rangle$ the duality of $V$ and its dual $V^{\prime}$ as well as the inner product on $H$, and by $\|\cdot\|,|\cdot|,\|\cdot\|_{V^{\prime}}$ the norms in $V, H$ and $V^{\prime}$, respectively. For a fixed real number $T>0$, we introduce the spaces $\mathcal{V}=L^{2}(0, T ; V), \mathcal{H}=L^{2}(0, T ; H)$ and $\mathcal{V}^{\prime}=L^{2}\left(0, T ; V^{\prime}\right)$. The duality between $\mathcal{V}$ and $\mathcal{V}^{\prime}$ and the inner product 
on $\mathcal{H}$ is denoted by

$$
\langle\langle f, v\rangle\rangle=\int_{0}^{T}\langle f(s), v(s)\rangle d s, \quad f \in \mathcal{V}^{\prime}, v \in \mathcal{V} .
$$

Moreover, given an open bounded subset $\Omega$ in $\mathbb{R}^{n}$ with Lipschitz continuous boundary $\Gamma$, we put $Q=\Omega \times(0, T)$ and $\Sigma=\Gamma \times(0, T)$. The duality between $L^{2}\left(0, T ; H^{1 / 2}(\Gamma)\right)$ and its dual (and the inner product on $L^{2}(\Sigma)$ ) is denoted by

$$
\langle\langle w, z\rangle\rangle_{\Sigma}=\int_{0}^{T}\langle w(s), z(s)\rangle_{\Gamma} d s,
$$

where $\langle\cdot, \cdot\rangle_{\Gamma}$ stands for the duality of $H^{1 / 2}(\Gamma)$ and its dual and also for the inner product on $L^{2}(\Gamma)$.

For a Banach space $\mathcal{X}$, the symbols $w-\mathcal{X}, s-\mathcal{X}$ are always used for the weak and the strong topology in $\mathcal{X}$, respectively. Given a sequence $v_{n} \in$ $L^{\infty}(0, T ; V)$, we will write $v_{n} \rightarrow v$ in $w-*-L^{\infty}(0, T ; V)$ if $\left\langle\left\langle v_{n}, g\right\rangle\right\rangle \rightarrow\langle\langle v, g\rangle\rangle$ as $n \rightarrow \infty$, for every $g \in L^{1}\left(0, T ; V^{\prime}\right)$. In particular, for $v_{n} \in L^{\infty}(0, T), v_{n} \rightarrow v$ in $w-*-L^{\infty}(0, T)$ means that $\int_{0}^{T} v_{n} g d t \rightarrow \int_{0}^{T} v g d t$ for all $g \in L^{1}(0, T)$. Given a convex function $f: \mathbb{R}^{d} \rightarrow \mathbb{R}$, we denote by $f^{*}: \mathbb{R}^{d} \rightarrow \mathbb{R}$ the polar (or conjugate) function of $f$, i.e. $f^{*}\left(z^{*}\right)=\sup \left\{z z^{*}-f(z) \mid z \in \mathbb{R}^{d}\right\}$. Different constants independent of the parameter $k$ are denoted by the same letter $c$. We also write $\overline{\mathbb{N}}=\mathbb{N} \cup\{\infty\}$. In what follows we use the standard summation convention.

We consider a family of linear operators $\mathcal{A}_{k}: \mathcal{V} \rightarrow \mathcal{V}^{\prime}, k \in \mathbb{N}$, of the form

$$
\mathcal{A}_{k}=-\frac{\partial}{\partial x_{i}}\left(a_{i j}^{k}(x, t) \frac{\partial}{\partial x_{j}}\right)
$$

with the coefficients $a_{i j}^{k} \in L^{\infty}(Q)$ which satisfy in $Q$, uniformly with respect to $k$, the following assumptions:

$$
\begin{gathered}
a_{i j}^{k}=a_{j i}^{k}, \\
\lambda_{0} \leq a_{i j}^{k} \xi_{i} \xi_{j}|\xi|^{-2} \leq \lambda_{1}, \quad \forall \xi \in \mathbb{R}^{n}, \\
\left|a_{i j}^{k}\left(x, t_{2}\right)-a_{i j}^{k}\left(x, t_{1}\right)\right| \leq M\left|t_{2}-t_{1}\right|
\end{gathered}
$$

for some real constants $\lambda_{0}, \lambda_{1}, M$ such that $0<\lambda_{0} \leq \lambda_{1}$ and $M>0$.

We denote by $H\left(\lambda_{0}, \lambda_{1}, M\right)$ the class of hyperbolic operators $H_{\mathcal{A}_{k}}=$ $\partial^{2} / \partial t^{2}+\mathcal{A}_{k}, H_{\mathcal{A}_{k}}: \mathcal{V} \supset \operatorname{dom}\left(H_{\mathcal{A}_{k}}\right) \rightarrow \mathcal{V}^{\prime}$ associated with operators $\mathcal{A}_{k}$ whose coefficients satisfy $(3)-(5)$. Let $\mathcal{E}\left(\lambda_{0}, \lambda_{1}\right)$ be the class of real measurable functions $a_{i j}^{k}, k \in \overline{\mathbb{N}}$, on $\Omega$ satisfying (3) and (4) uniformly with respect to $k$.

Following [18], [7], [20], we make the following 
Definition 2.1. We say that a sequence $\left(a_{i j}^{k}\right) \in \mathcal{E}\left(\lambda_{0}, \lambda_{1}\right) G$-converges to $a_{i j}^{\infty}$ on $\Omega$ as $k \rightarrow \infty$ (and write $a_{i j}^{k} \stackrel{G}{\rightarrow} a_{i j}^{\infty}$ on $\Omega$ ) iff for every $f \in H^{-1}(\Omega)$, we have $u_{k} \rightarrow u_{\infty}$ weakly in $H_{0}^{1}(\Omega)$, where $u_{k}, k \in \overline{\mathbb{N}}$, denotes the solution of the problem

$$
\left\{\begin{array}{l}
-\frac{\partial}{\partial x_{i}}\left(a_{i j}^{k}(x) \frac{\partial u_{k}}{\partial x_{j}}\right)=f \quad \text { in } \Omega, \\
u_{k} \in H_{0}^{1}(\Omega) .
\end{array}\right.
$$

For the definition and properties of $G$-convergence in the abstract case see $[18]-[20]$.

For the reader's convenience, let us also recall the notion of sequential $\Gamma$-convergence for function(al)s of two variables. The case of one variable is trivial since it suffices to omit the other variable. Let $\mathcal{X}_{i}, i=1,2$, be topological spaces, let $x_{i} \in \mathcal{X}_{i}$ and $S_{i}=S\left(x_{i}\right)=\left\{\left(x_{i}^{k}\right) \subset \mathcal{X}_{i} \mid x_{i}^{k} \rightarrow x_{i}\right.$ in $\mathcal{X}_{i}$ as $k \rightarrow \infty\}$. Given the functionals $J_{k}: \mathcal{X}_{1} \times \mathcal{X}_{2} \rightarrow \overline{\mathbb{R}}, k \in \overline{\mathbb{N}}$, we adopt

Definition 2.2. We say that the sequence $J_{k}$ is $\Gamma_{\text {seq }}\left(\mathcal{X}_{1}^{-}, \mathcal{X}_{2}\right)$-convergent to $J_{\infty}\left(\right.$ and write $\left.J_{\infty}=\Gamma_{\text {seq }}\left(\mathcal{X}_{1}^{-}, \mathcal{X}_{2}\right) \lim J_{k}\right)$ iff for every $\left(x_{1}, x_{2}\right) \in \mathcal{X}_{1} \times \mathcal{X}_{2}$, the four extended-real numbers

$$
\begin{aligned}
& \Gamma_{\text {seq }}\left(\mathcal{X}_{1}^{-}, \mathcal{X}_{2}^{-}\right) \liminf J_{k}\left(x_{1}, x_{2}\right)=\inf _{S_{1}} \inf _{S_{2}} \liminf _{k \rightarrow \infty} J_{k}\left(x_{1}^{k}, x_{2}^{k}\right), \\
& \Gamma_{\text {seq }}\left(\mathcal{X}_{1}^{-}, \mathcal{X}_{2}^{-}\right) \limsup J_{k}\left(x_{1}, x_{2}\right)=\inf _{S_{1}} \inf _{S_{2}} \limsup _{k \rightarrow \infty} J_{k}\left(x_{1}^{k}, x_{2}^{k}\right), \\
& \Gamma_{\text {seq }}\left(\mathcal{X}_{1}^{-}, \mathcal{X}_{2}^{+}\right) \liminf J_{k}\left(x_{1}, x_{2}\right)=\inf _{S_{1}} \sup _{S_{2}} \liminf _{k \rightarrow \infty} J_{k}\left(x_{1}^{k}, x_{2}^{k}\right), \\
& \Gamma_{\text {seq }}\left(\mathcal{X}_{1}^{-}, \mathcal{X}_{2}^{+}\right) \limsup J_{k}\left(x_{1}, x_{2}\right)=\inf _{S_{1}} \sup _{S_{2}} \limsup _{k \rightarrow \infty} J_{k}\left(x_{1}^{k}, x_{2}^{k}\right)
\end{aligned}
$$

are equal to $J_{\infty}\left(x_{1}, x_{2}\right)$.

In the sequel, since we only use the sequential $\Gamma$-convergence, we shall omit the subscript "seq" appearing in the above definition.

Remark 2.1. If $\mathcal{X}$ is a topological space and $f_{k}: \mathcal{X} \rightarrow \overline{\mathbb{R}}$ satisfy

(i) for every $x \in \mathcal{X}$ and every sequence $x_{k} \rightarrow x$,

$$
f_{\infty}(x) \leq \liminf _{k \rightarrow \infty} f_{k}\left(x_{k}\right)
$$

(ii) for every $x \in \mathcal{X}$, there exists a sequence $x_{k} \rightarrow x$ such that

$$
f_{\infty}(x)=\lim _{k \rightarrow \infty} f_{k}\left(x_{k}\right)
$$

then $f_{\infty}=\Gamma\left(\mathcal{X}^{-}\right) \lim f_{k}$.

The property which motivates the introduction of $\Gamma$-convergence in the calculus of variations is the following 
Proposition 2.1. Let $f_{k}: \mathcal{X} \rightarrow \overline{\mathbb{R}}$ and $f_{\infty}=\Gamma\left(\mathcal{X}^{-}\right) \lim f_{k}$. Assume that there exists a sequence $\left(\widehat{x}_{k}\right) \subset \mathcal{X}$ such that $\widehat{x}_{k} \rightarrow \widehat{x}$ and

$$
\liminf _{k \rightarrow \infty} f_{k}\left(\widehat{x}_{k}\right)=\liminf _{k \rightarrow \infty}\left(\inf _{\mathcal{X}} f_{k}\right) .
$$

Then

$$
f_{\infty}(\widehat{x})=\min _{\mathcal{X}} f_{\infty}=\lim _{k \rightarrow \infty}\left(\inf _{\mathcal{X}} f_{k}\right) .
$$

For further information on $\Gamma$-convergence we refer to [5], [6] and the references cited there.

3. Main result. Consider first the initial value problem

$$
\begin{cases}H_{\mathcal{A}} y=f & \text { in } Q, \\ \partial y / \partial \nu_{\mathcal{A}}=v & \text { on } \Sigma, \\ y(0)=\phi & \text { in } \Omega, \\ y^{\prime}(0)=\psi & \text { in } \Omega,\end{cases}
$$

where $\partial / \partial \nu_{\mathcal{A}}=a_{i j}(x, t) \cos \left(\nu, x_{i}\right) \partial / \partial x_{j}$ denotes the outward conormal derivative on $\Gamma$ corresponding to the coefficients $a_{i j}(x, t)$ (see e.g. [11]). As mentioned earlier, in order to study (7), we use the method of transposition (cf. [11] or [9]). To this end, taking $V=H^{1}(\Omega), H=L^{2}(\Omega)$, we recall (see Theorem 1.1 in Chapter IV of [9]) that given $\bar{f} \in \mathcal{H}$, there exists a unique $z \in \mathcal{V}$ with $z^{\prime} \in \mathcal{H}$ satisfying

$$
\begin{cases}H_{\mathcal{A}} z=\bar{f} & \text { in } Q, \\ \partial z / \partial \nu_{\mathcal{A}}=0 & \text { on } \Sigma, \\ z(T)=0 & \text { in } \Omega, \\ z^{\prime}(T)=0 & \text { in } \Omega .\end{cases}
$$

Let $\mathcal{Z}$ denote the space spanned by $z$ as $\bar{f}$ ranges over $\mathcal{H}$.

Definition 3.1 A function $y \in \mathcal{H}$ is called a weak solution to (7) iff

$$
\left\langle\left\langle y, H_{\mathcal{A}} z\right\rangle\right\rangle=\langle\langle f, z\rangle\rangle-\left\langle\phi, z^{\prime}(0)\right\rangle+\langle\psi, z(0)\rangle+\langle\langle v, z\rangle\rangle_{\Sigma}
$$

for every $z \in \mathcal{Z}$.

It is well known (see Chapter IV of [9]) that for every $H_{\mathcal{A}} \in H\left(\lambda_{0}, \lambda_{1}, M\right)$, $f \in \mathcal{H}, v \in L^{2}(\Sigma), \phi \in H, \psi \in V^{\prime}$ there exists a unique weak solution to (7) in the sense of Definition 3.1. Moreover, this solution depends continuously on the data $f, v, \phi, \psi$.

Now, given a sequence of operators $\left(H_{\mathcal{A}_{k}}\right), k \in \overline{\mathbb{N}}$, of class $H\left(\lambda_{0}, \lambda_{1}, M\right)$, consider the family of initial value problems (the state equations in control problems):

$$
\begin{cases}H_{\mathcal{A}_{k}} y=f & \text { in } Q, \\ \partial y / \partial \nu_{\mathcal{A}_{k}}=v & \text { on } \Sigma, \\ y(0)=\phi & \text { in } \Omega, \\ y^{\prime}(0)=\psi & \text { in } \Omega .\end{cases}
$$


We define the sets of admissible control-state pairs by

$$
\Lambda_{k}=\left\{(u, y) \in U \times Y \mid(u, y) \text { satisfies }(8)_{k}\right\},
$$

where $u=(f, v, \phi, \psi), U=\mathcal{H} \times L^{2}(\Sigma) \times V \times H$ and $Y=\mathcal{H}$.

We study the sequence of control problems

$$
\min \left\{J_{k}(u, y)+\chi_{\Lambda_{k}}(u, y) \mid(u, y) \in U \times Y\right\}, \quad k \in \overline{\mathbb{N}} .
$$

The cost functionals in $(10)_{k}$ are given by

(11) $J_{k}((f, v, \phi, \psi), y)=J_{k}^{(1)}(f, y)+J_{k}^{(2)}(v)+J_{k}^{(3)}(\phi)+J_{k}^{(4)}(\psi), \quad k \in \overline{\mathbb{N}}$, where

$$
\begin{aligned}
J_{k}^{(1)}(f, y) & =\int_{Q} F_{k}^{(1)}(x, t, y(x, t), f(x, t)) d x d t, \quad(f, y) \in \mathcal{H} \times \mathcal{H}, \\
J_{k}^{(2)}(v) & =\int_{\Sigma} F_{k}^{(2)}(x, t, v(x, t)) d \sigma d t, \quad v \in L^{2}(\Sigma), \\
J_{k}^{(3)}(\phi) & =\int_{\Omega} F_{k}^{(3)}(x, \phi(x), \nabla \phi(x)) d x, \quad \phi \in V, \\
J_{k}^{(4)}(\psi) & =\int_{\Omega} F_{k}^{(4)}(x, \psi(x)) d x, \quad \psi \in H .
\end{aligned}
$$

We need the following hypotheses on the integrands $F_{k}^{(i)}, i=1,2,3,4, k \in \overline{\mathbb{N}}$ $\left(F_{k}^{(i) *}\right.$ denotes the polar function to $F_{k}^{(i)}$ with respect to the starred variables):

$\left(\mathrm{H}_{1}\right) \quad$ (a) $F_{k}^{(1)}: Q \times \mathbb{R}^{2} \rightarrow \mathbb{R}$ are Borel functions and $F_{k}^{(1)}(x, t, y, \cdot)$ are convex for all $(x, t) \in Q$ and $y \in \mathbb{R}$,

(b) there exists $c \geq 1$ such that

$$
|z|^{2} \leq F_{k}^{(1)}(x, t, y, z) \leq c\left(1+|y|^{2}+|z|^{2}\right)
$$

(c) $\left|F_{k}^{(1)}(x, t, y, z)-F_{k}^{(1)}\left(x, t, y_{1}, z\right)\right| \leq \varrho\left(\left|y-y_{1}\right|\right)\left(1+|y|^{2}+|z|^{2}\right)$ for $(x, t) \in Q, z \in \mathbb{R}$, and $y, y_{1} \in \mathbb{R}$ such that $\left|y-y_{1}\right| \leq 1$, and $\varrho:[0,1) \rightarrow \mathbb{R}$ is a continuous, increasing function with $\varrho(0)=0$,

(d) $F_{k}^{(1) *}\left(\cdot, \cdot, y, z^{*}\right) \rightarrow F_{\infty}^{(1) *}\left(\cdot, \cdot, y, z^{*}\right)$ in $w-L^{1}(Q)$ as $k \rightarrow \infty$, for all $y, z^{*} \in \mathbb{R}$

$\left(\mathrm{H}_{2}\right) \quad$ (a) $F_{k}^{(2)}: \Sigma \times \mathbb{R} \rightarrow \mathbb{R}$ are Borel functions, $F_{k}^{(2)}(x, t, \cdot)$ are convex for all $(x, t) \in \Sigma$,

(b) there exists $c>0$ such that $c|z|^{2} \leq F_{k}^{(2)}(x, t, z)$ for $(x, t) \in \Sigma$ and $z \in \mathbb{R}$,

(c) $F_{k}^{(2) *}\left(\cdot, \cdot, z^{*}\right) \rightarrow F_{\infty}^{(2) *}\left(\cdot, \cdot, z^{*}\right)$ in $w-*-L^{\infty}(\Sigma)$ as $k \rightarrow \infty$, for all $z^{*} \in \mathbb{R}$ 
$\left(\mathrm{H}_{3}\right) \quad\left(\right.$ a) $F_{k}^{(3)}: \Omega \times \mathbb{R}^{n+1} \rightarrow \mathbb{R}$ are Borel functions, $F_{k}^{(3)}(x, \cdot)$ are convex for all $x \in \Omega$,

(b) $c_{1}|z|^{2} \leq F_{k}^{(3)}(x, z) \leq c_{2}|z|^{2}$, with $0<c_{1} \leq c_{2}$, for all $x \in \Omega$ and $z \in \mathbb{R}^{n+2}$,

(c) both $F_{k}^{(3)}(\cdot, z) \rightarrow F_{\infty}^{(3)}(\cdot, z)$ and $F_{k}^{(3) *}\left(\cdot, z^{*}\right) \rightarrow F_{\infty}^{(3) *}\left(\cdot, z^{*}\right)$ in $w-*-L^{\infty}(\Omega)$ as $k \rightarrow \infty$ for all $z, z^{*} \in \mathbb{R}^{n+1}$;

$\left(\mathrm{H}_{4}\right) \quad\left(\right.$ a) $F_{k}^{(4)}: \Omega \times \mathbb{R} \rightarrow \mathbb{R}$ are Borel functions, $F_{k}^{(4)}(x, \cdot)$ are convex for all $x \in \Omega$,

(b) there exists $c>0$ such that $c|v|^{2} \leq F_{k}^{(4)}(x, v)$ for $x \in \Omega$ and $v \in \mathbb{R}$,

(c) $F_{k}^{(4) *}\left(\cdot, v^{*}\right) \rightarrow F_{\infty}^{(4) *}\left(\cdot, v^{*}\right)$ in $w-*-L^{\infty}(\Omega)$ as $k \rightarrow \infty$ for all $v^{*} \in$ $\mathbb{R}$.

As regards the hyperbolic operators, we assume that

$\left(\mathrm{H}_{5}\right) \quad$ (a) $H_{\mathcal{A}_{k}} \in H\left(\lambda_{0}, \lambda_{1}, M\right)$ for $k \in \overline{\mathbb{N}}$,

(b) $a_{i j}^{k}(\cdot, t) \stackrel{G}{\rightarrow} a_{i j}^{\infty}(\cdot, t)$ on $\Omega$, for all $t \in[0, T]$, as $k \rightarrow \infty$.

We have the following

TheOREM 3.1. (i) If hypotheses (a), (b), (c) of $\left(\mathrm{H}_{1}\right)$, (a), (b) of $\left(\mathrm{H}_{2}\right)$, (a), (b) of $\left(\mathrm{H}_{3}\right)$, (a), (b) of $\left(\mathrm{H}_{4}\right)$ and $(\mathrm{a})$ of $\left(\mathrm{H}_{5}\right)$ hold, then for every fixed $k \in \overline{\mathbb{N}}$, there exists an optimal solution $\left(\widetilde{u}_{k}, \widetilde{y}_{k}\right) \in U \times Y$ to control problem $(10)_{k}$ with $J_{k}$ defined by $(11)$.

(ii) If hypotheses $\left(\mathrm{H}_{1}\right)-\left(\mathrm{H}_{5}\right)$ are satisfied, then the sequence $\left(\widetilde{u}_{k}, \widetilde{y}_{k}\right)$ has a subsequence converging in $(w-U) \times(s-Y)$ topology to an optimal solution $\left(\widetilde{u}_{\infty}, \widetilde{y}_{\infty}\right)$ of the limit problem $(10)_{\infty}$. Moreover, the sequence of minimal values $\left(J_{k}\left(\widetilde{u}_{k}, \widetilde{y}_{k}\right)\right)$ converges to the minimal value $J_{\infty}\left(\widetilde{u}_{\infty}, \widetilde{y}_{\infty}\right)$.

(iii) If the limit problem has a unique optimal solution, then the sequence $\left(\widetilde{u}_{k}, \widetilde{y}_{k}\right)$ itself converges to $\left(\widetilde{u}_{\infty}, \widetilde{y}_{\infty}\right)$.

4. Proof of the main result. The proof of Theorem 3.1 is based on the following abstract result concerning the asymptotic behaviour of $(1)_{k}$ and obtained in [2] as the sequential version of Proposition 2.1.

Proposition 4.1. Let $U, Y$ be topological spaces and let $\left(u_{k}, y_{k}\right)$ be optimal (or quasi-optimal in the sense of (6)) solutions to $(1)_{k}, k \in \overline{\mathbb{N}}$, such that $\left(u_{k}, y_{k}\right) \rightarrow\left(u_{\infty}, y_{\infty}\right)$ in $U \times Y$. Let

$$
\begin{aligned}
J_{\infty} & =\Gamma\left(U^{-}, Y\right) \lim J_{k}, \\
\chi_{\Lambda_{\infty}} & =\Gamma\left(U, Y^{-}\right) \lim \chi_{\Lambda_{k}} .
\end{aligned}
$$

Then $\left(u_{\infty}, y_{\infty}\right)$ is an optimal solution to $(1)_{\infty}$. 
We first remark (following [6], [2], [8]) that for $\Lambda_{k} \subset U \times Y, k \in \mathbb{N}$, the $\Gamma$-limit of the indicator function of $\Lambda_{k}$ is also the indicator function of a set $\Lambda_{\infty}$ and (13) is equivalent to the following two conditions:

(14) if $u_{k} \rightarrow u_{\infty}, y_{k} \rightarrow y_{\infty}$ and $\left(u_{k}, y_{k}\right) \in \Lambda_{k}$ for infinitely many $k$ then $\left(u_{\infty}, y_{\infty}\right) \in \Lambda_{\infty}$

(15) if $u_{k} \rightarrow u_{\infty},\left(u_{\infty}, y_{\infty}\right) \in \Lambda_{\infty}$ then there are $y_{k} \rightarrow y_{\infty}$ and $k_{0} \in \mathbb{N}$ such that $\left(u_{k}, y_{k}\right) \in \Lambda_{k}$ for $k \geq k_{0}$.

Next, in order to apply Proposition 4.1, we will show two lemmas which elaborate on conditions (12) and (14), (15) in the case when $\Lambda_{k}$ and $J_{k}$ are defined by (9) and (11), respectively.

LEMMA 4.1. If hypotheses $\left(\mathrm{H}_{1}\right)-\left(\mathrm{H}_{4}\right)$ hold, then

$$
J_{\infty}=\Gamma\left(w-U^{-}, s-Y\right) \lim J_{k},
$$

where $J_{k}, k \in \overline{\mathbb{N}}$, are defined by (11).

Proof. First of all, we can show that the functionals $J_{k}^{(i)}$ are $\Gamma$-convergent to $J_{\infty}^{(i)}, i=1,2,3,4$. Namely adding $t$ to the independent variable $x$ and augmenting the space variable from $\mathbb{R}$ to $\mathbb{R}^{2}$ in Lemma 3.1 of [2] and Theorem 3.4 of [12], we get

$$
J_{\infty}^{(1)}=\Gamma\left(w-\mathcal{H}^{-}, s-\mathcal{H}\right) \lim J_{k}^{(1)}, \quad J_{\infty}^{(2)}=\Gamma\left(w-L^{2}(\Sigma)^{-}\right) \lim J_{k}^{(2)},
$$

respectively. Under our assumptions, from Theorems 3.3 and 3.4 of [12] we have (see also [8])

$$
J_{\infty}^{(3)}=\Gamma\left(w-V^{-}\right) \lim J_{k}^{(3)} .
$$

Again, by Theorem 3.4 of [12], we directly deduce that

$$
J_{\infty}^{(4)}=\Gamma\left(w-H^{-}\right) \lim J_{k}^{(4)} .
$$

Now, using Lemma 2.8 of [14], we calculate the sum of these four $\Gamma$-limits and hence we obtain the result.

Let $\left(f_{k}, v_{k}, \phi_{k}, \psi_{k}\right) \in \mathcal{H} \times L^{2}(\Sigma) \times H \times V^{\prime}, k \in \overline{\mathbb{N}}$, and suppose hypothesis $\left(\mathrm{H}_{5}\right)$ (a) holds. We denote by $y_{k} \in \mathcal{H}, k \in \overline{\mathbb{N}}$, the unique solutions (in the sense of Definition 3.1) of the problem $(8)_{k}$ with right hand sides $f_{k}, v_{k}, \phi_{k}$ and $\psi_{k}$.

LemMA 4.2. If hypothesis $\left(\mathrm{H}_{5}\right)$ holds and

$$
\begin{array}{lll}
f_{k} \rightarrow f_{\infty} & \text { in } w-\mathcal{H}, & \phi_{k} \rightarrow \phi_{\infty} \quad \text { in } s-H, \\
v_{k} \rightarrow v_{\infty} & \text { in } w-L^{2}(\Sigma), & \psi_{k} \rightarrow \psi_{\infty} \quad \text { in } s-V^{\prime},
\end{array}
$$

as $k \rightarrow \infty$, then

$$
y_{k} \rightarrow y_{\infty} \quad \text { in } s-\mathcal{H}
$$


where $y_{\infty}$ is the solution (unique in the same sense) of the problem $(8)_{\infty}$ corresponding to $f_{\infty}, v_{\infty}, \phi_{\infty}$ and $\psi_{\infty}$.

Proof. From the hypotheses and from the uniform a priori estimate (see $[9]$ )

$$
\left\|y_{k}\right\|_{\mathcal{H}} \leq c\left(\left\|f_{k}\right\|_{\mathcal{H}}+\left\|v_{k}\right\|_{L^{2}(\Sigma)}+\left|\phi_{k}\right|+\left\|\psi_{k}\right\|_{V^{\prime}}\right)
$$

where $c$ is independent of $k$, we deduce that $\left(y_{k}\right)$ lies in a bounded subset of $\mathcal{H}$. Therefore passing to a subsequence if necessary, again called $y_{k}$, we may assume

$$
y_{k} \rightarrow y_{0} \quad \text { in } w-\mathcal{H}
$$

with some $y_{0} \in \mathcal{H}$. In what follows, we shall show that $y_{0}=y_{\infty}$.

To this end, fix $\bar{f} \in \mathcal{H}$. By definition, $y_{k}$ satisfies the equality

$$
\left\langle\left\langle y_{k}, H_{\mathcal{A}_{k}} z_{k}\right\rangle\right\rangle=\left\langle\left\langle f_{k}, z_{k}\right\rangle\right\rangle-\left\langle\phi_{k}, z_{k}^{\prime}(0)\right\rangle+\left\langle\psi_{k}, z_{k}(0)\right\rangle+\left\langle\left\langle v_{k}, z_{k}\right\rangle\right\rangle_{\Sigma},
$$

where $z_{k} \in \mathcal{V}, k \in \mathbb{N}$, is the solution to

$$
\begin{cases}H_{\mathcal{A}_{k}} z_{k}=\bar{f} & \text { in } Q, \\ \partial z_{k} / \partial \nu_{\mathcal{A}_{k}}=0 & \text { on } \Sigma, \\ z_{k}(T)=0 & \text { in } \Omega, \\ z_{k}^{\prime}(T)=0 & \text { in } \Omega .\end{cases}
$$

Now, by hypothesis $\left(\mathrm{H}_{5}\right)$, from Lemma 2.2 of [13] (see also [4]), we have

$$
\begin{array}{ll}
z_{k} \rightarrow z_{\infty} & \text { in } w-*-L^{\infty}(0, T ; V) \text { and in } s-C([0, T] ; H), \\
z_{k}^{\prime} \rightarrow z_{\infty}^{\prime} & \text { in } w-*-L^{\infty}(0, T ; H) \text { and in } s-C\left([0, T] ; V^{\prime}\right),
\end{array}
$$

where $z_{\infty}$ is a solution of the limit problem $(21)_{\infty}$.

Since $\left(z_{k}\right)$ and $\left(z_{k}^{\prime}\right)$ are bounded in $\mathcal{V}$ and $\mathcal{H}$, respectively, according to a well known compactness theorem (see e.g. [11], [9]), $\left(z_{k}\right)$ is a precompact subset of some $L^{2}\left(0, T ; H^{\beta}(\Omega)\right)$, where $\beta \in(1 / 2,1)$. Thus by the trace theorem we conclude that $\left(\left.z_{k}\right|_{\Sigma}\right)$ is precompact in $L^{2}(\Sigma)$. Hence without loss of generality, we can suppose that

$$
\left.\left.z_{k}\right|_{\Sigma} \rightarrow z_{\infty}\right|_{\Sigma} \quad \text { in } s-L^{2}(\Sigma)
$$

By (16), (19), (22) and (23) we can pass to the limit in (20) as $k \rightarrow \infty$ to get

(24) $\left\langle\left\langle y_{0}, \bar{f}\right\rangle\right\rangle=\left\langle\left\langle f_{\infty}, z_{\infty}\right\rangle\right\rangle-\left\langle\phi_{\infty}, z_{\infty}^{\prime}(0)\right\rangle+\left\langle\psi_{\infty}, z_{\infty}(0)\right\rangle+\left\langle\left\langle v_{\infty}, z_{\infty}\right\rangle_{\Sigma}\right.$.

Taking into account that $z_{\infty}$ satisfies $(21)_{\infty}$, we conclude from the arbitrariness of $\bar{f}$ that $y_{0}$ is a weak solution of $(8)_{\infty}$ corresponding to $f_{\infty}, v_{\infty}, \phi_{\infty}$ and $\psi_{\infty}$. By the uniqueness of solutions to this problem we get $y_{0}=y_{\infty}$ and $y_{k} \rightarrow y_{\infty}$ in $w-\mathcal{H}$. To conclude, it is enough to show that the last convergence is strong. Putting in $(21)_{k}, \bar{f}=y_{k}$ and $\bar{f}=y_{\infty}$, respectively, and 
using (24), a short computation gives

$$
\begin{aligned}
\left\|y_{k}-y_{\infty}\right\|_{\mathcal{H}}^{2}= & \left\langle\left\langle f_{\infty}, z_{\infty}\right\rangle\right\rangle-\left\langle\left\langle f_{k}, z_{k}\right\rangle\right\rangle+\left\langle\psi_{\infty}, z_{\infty}(0)\right\rangle-\left\langle\psi_{k}, z_{k}(0)\right\rangle \\
& +\left\langle\phi_{k}, z_{k}^{\prime}(0)\right\rangle-\left\langle\phi_{\infty}, z_{\infty}^{\prime}(0)\right\rangle+\left\langle\left\langle v_{\infty}, z_{\infty}\right\rangle\right\rangle_{\Sigma}-\left\langle\left\langle v_{k}, z_{k}\right\rangle\right\rangle_{\Sigma} .
\end{aligned}
$$

From (16), (22) and (23) it follows that each term on the right hand side tends to zero as $k \rightarrow \infty$, showing (17).

Proof of Theorem 3.1. For the proof of assertion (i) of Theorem 3.1 we apply the direct method. Fix $k \in \overline{\mathbb{N}}$ and let $\left\{\left(u_{n}, y_{n}\right)\right\}$ be a minimizing sequence in $U \times Y$, i.e.

$$
J_{k}\left(u_{n}, y_{n}\right) \rightarrow \inf \left\{J_{k}(u, y) \mid(u, y) \in \Lambda_{k}\right\}
$$

where $u_{n}=\left(f_{n}, v_{n}, \phi_{n}, \psi_{n}\right)$. Note that from assumptions $\left(\mathrm{H}_{1}\right)(\mathrm{b}),\left(\mathrm{H}_{2}\right)(\mathrm{b})$, $\left(\mathrm{H}_{3}\right)(\mathrm{b}),\left(\mathrm{H}_{4}\right)(\mathrm{b})$ we have that $\left\|u_{n}\right\|_{U} \leq c$, where $U=\mathcal{H} \times L^{2}(\Sigma) \times V \times H$ and $c$ is independent of $n$. Next, in view of the compactness of the embeddings $V \subset H \subset V^{\prime}$ and from the reasoning analogous to that in Lemma 4.2, we deduce that $\left\{\left(u_{n}, y_{n}\right)\right\}$ is compact in $(w-U) \times(s-Y)$ topology. Since the $\Gamma$-limit of a constant sequence of functionals gives the l.s.c. envelope of the functional (see e.g. [6], [5], [8]), for every fixed $k \in \overline{\mathbb{N}}$, we have the sequential l.s.c. of $J_{k}$ in the same topology. This completes the proof of (i).

Proof of (ii). As above, we find that the sequence of optimal solutions to $(10)_{k}$ is compact in $(w-U) \times(s-Y)$ topology. Furthermore, from Lemma 4.2 and from the compactness of the embedding $V \subset H$, we find that the conditions (14) and (15) hold for $\Lambda_{k}$ defined by (9). This proves that

$$
\chi_{\Lambda_{\infty}}=\Gamma\left(w-U, s-Y^{-}\right) \lim \chi_{\Lambda_{k}} .
$$

From this relation and from Lemma 4.1, it follows that we may apply Proposition 4.1, which in turn immediately implies the assertion (ii) of the theorem. The convergence of the minimal values is a consequence of Proposition 2.1. Finally, (iii) follows directly from (i) and (ii).

Acknowledgements. The author would like to thank Professor Z. Denkowski for helpful conversations and the referee for his (her) remarks.

\section{References}

[1] A. Bensoussan, J. L. Lions and G. Papanicolaou, Asymptotic Analysis for Periodic Structures, Stud. Math. Appl. 5, North-Holland, Amsterdam, 1978.

[2] G. Buttazzo and G. Dal Maso, $\Gamma$-convergence and optimal control problems, J. Optim. Theory. Appl. 38 (1982), 385-407.

[3] F. Colombini et S. Spagnolo, Sur la convergence de solutions d'équations paraboliques, J. Math. Pures Appl. 56 (1977), 263-306. 
[4] F. Colombini et S. Spagnolo, On convergence of solutions of hyperbolic equations, Comm. Partial Differential Equations 3 (1978), 77-91.

[5] E. De Giorgi, Convergence problems for functionals and operators, in: Proc. Internat. Meeting on Recent Methods in Nonlinear Analysis, E. De Giorgi, E. Magenes and U. Mosco (eds.), Pitagora, Bologna, 1979, 131-188.

[6] E. De Giorgi e T. Franzoni, Su un tipo di convergenza variazionale, Rend. Sem. Mat. Brescia 3 (1979), 63-101.

[7] E. De Giorgi e S. Spagnolo, Sulla convergenza degli integrali della energia per operatori ellitici del secondo ordine, Boll. Un. Mat. Ital. 8 (1973), 391-411.

[8] Z. Denkowski and S. Migórski, Control problems for parabolic and hyperbolic equations via the theory of $G$ and $\Gamma$ convergence, Ann. Mat. Pura Appl. (4) 149 (1987), 23-39.

[9] J. L. Lions, Optimal Control of Systems Governed by Partial Differential Equations, Springer, Berlin, 1971.

[10] - Some Methods in the Mathematical Analysis of Systems and their Control, Science Press, Beijing and Gordon and Breach, New York, 1981.

[11] J. L. Lions and E. Magenes, Non-Homogeneous Boundary-Value Problems, Vol. I, Springer, Berlin, 1972.

[12] P. Marcellini e C. Sbordone, Dualità e perturbazione di funzionali integrali, Ricerche Mat. 26 (1977), 383-421.

[13] S. Migórski, Convergence of optimal solutions in control problems for hyperbolic equations, preprint CSJU 1/1991, Jagiellonian University, Kraków.

[14] —, Asymptotic behaviour of optimal solutions in control problems for elliptic equations, Riv. Mat. Pura Appl. 11 (1992), 7-28.

[15] - On asymptotic limits of control problems with parabolic and hyperbolic equations, ibid. 12 (1992), 33-50.

[16] - Sensitivity analysis of distributed parameter optimal control problems for nonlinear parabolic equations, J. Optim. Theory Appl. 87 (1995), to appear.

[17] E. Sanchez-Palencia, Nonhomogeneous Media and Vibration Theory, Lecture Notes in Phys. 127, Springer, Berlin, 1980.

[18] S. Spagnolo, Sulla convergenza di equazioni paraboliche ed ellittiche, Ann. Scuola Norm. Sup. Pisa Cl. Sci. (3) 22 (1968), 577-597.

[19] - Convergence in energy for elliptic operators, in: Proc. Third Symp. Numer. Solutions PDE (College Park, 1975), Academic Press, San Diego, 1976, 469-498.

[20] V. V. Zhikov, S. M. Kozlov, O. A. Oleŭnik and Kha T'en Ngoan, Averaging and G-convergence of differential operators, Russian Math. Surveys 34 (1979), 69147.

INSTITUTE OF COMPUTER SCIENCE

JAGIELLONIAN UNIVERSITY

NAWOJKI 11

30-072 KRAKÓW, POLAND 